\title{
Revealing treacherous points for successful light-front phenomenological applications
}

\author{
Chueng-Ryong Ji ${ }^{\mathrm{a} *}$, Bernard L. G. Bakker ${ }^{\mathrm{b}}$ and Ho-Meoyng Choi ${ }^{\dagger} \dagger$ \\ a Department of Physics, North Carolina State University, \\ Raleigh, NC 27695-8202, USA \\ ${ }^{\mathrm{b}}$ Department of Physics and Astronomy, Vrije Universiteit, \\ De Boelelaan 1081, NL-1081 HV Amsterdam, The Netherlands \\ ${ }^{\mathrm{c} D e p a r t m e n t}$ of Physics, Kyungpook National University, \\ Daegu 702-701, Korea
}

Light-front dynamics (LFD) plays an important role in hadron phenomenology as evidenced from recent development of generalized parton distributions and other physical quantities involving hadrons. For successful LFD applications to hadron phenomenology, however, treacherous points such as zero-mode contributions should be taken into account. For a concrete example of zero-mode contribution, we present Standard Model analysis of vector anomaly in the CP-even form factors of $W^{ \pm}$gauge bosons. The main distinguished features of LFD are discussed in comparison with other Hamiltonian dynamics. We also present a power counting method to correctly pin down which hadron form factors receive the zero-mode contribution and which ones do not. Indications from our analysis to hadron phenomenology are discussed.

\section{Introduction}

Light-front dynamics (LFD) provides a unified framework to analyze various experimental measurements such as generalized parton distributions (GPDs) and single spin asymmetry (SSA) at JLab and DESY (Hermes) [1, B-decays at SLAC (BaBar) and KEK (Belle) 2] as well as quark gluon plasma (QGP) productions at BNL (RHIC) and CERN (ALICE) 3, etc.. Due to the rational energy-momentum dispersion relation, the LFD has distinguished features compared to other forms of Hamiltonian dynamics. In particular, the vacuum fluctuations are suppressed and the kinematic generators are proliferated in LFD. Overall, these distinguished features can be regarded as advantageous rather than as disadvantageous in the hadron phenomenology. However, in return, the LFD implies treacher-

\footnotetext{
*Supported by Department of Energy under the contract DE-FG02-96ER40947.

${ }^{\dagger}$ Supported by Korean Research Foundation under the contract KRF-2005-070-C00039.
}

ous points, an example of which one may realize from the significance of zero-mode contributions even in the good $(+)$ current analyses [4]. Moreover, it has been shown that the common belief of equivalence between the manifestly covariant calculation and the light-front (LF) calculation is not always realized [5] unless treacherous points are well taken care of [6]. Thus, careful investigations of treacherous points and judicious ways of handling those points should be precedent for LFD to be distinctively useful compared to other forms of Hamiltonian dynamics [7].

In this presentation, we discuss a concrete example of a zero-mode contribution found in the Standard Model analysis of the vector anomaly in CP-even form factors of $W^{ \pm}$gauge bosons. No model dependences are involved in this discussion except that the analysis is made in the Standard Model. We then extend our discussion to hadron phenomenology.

In hadron phenomenology, observations of zero-mode contribution have been made by many 
authors 489101112 for the electroweak form factors involving a spin- 1 particle such as $\rho$ meson or deuteron. In particular, Jaus [8] proposed a covariant LF approach involving a lightlike fourvector $\omega^{\mu}\left(\omega^{2}=0\right)$ as a variable and developed a way of finding zero-mode contributions to remove spurious amplitudes proportional to $\omega^{\mu}$, while our method of finding zero-mode contributions is a direct power counting of the longitudinal momentum fraction for the off-diagonal elements in the Fock-state expansion of the current matrix 491011]. However, Jaus [8] and we 491011 do not agree on which form factors receive zero-mode contributions when the vector meson vertex $\Gamma^{\mu}$ is extended to more phenomenologically accessible ones. Utilizing manifestly covariant models for the vector meson vertex $\Gamma^{\mu}$, we find that Jaus's method of finding zero-mode contributions has a limitation for the choice of $\Gamma^{\mu}$ 1011. In the absence of zero-mode contributions, the hadron form factors can be obtained by just taking into account only the valence contributions (or diagonal matrix elements in the LF Fock-state expansion). Thus, it is quite significant in hadron phenomenology to correctly pin down which form factors receive the zero-mode contribution and which ones do not.

In the next Section, Section 2, we begin with a brief review on distinguished features of LFD in comparison with other forms of Hamiltonian dynamics. In Section 3, we present the zeromode contribution in Standard Model analysis of vector anomaly in CP-even form factors of $W^{ \pm}$ gauge bosons. In Section 4, we discuss our powercounting method to pin down zero-mode contributions. Conclusions follow in Section 5 .

\section{Distinguished Features of LFD}

Among the three forms of Hamiltonian dynamics proposed by Dirac in 1949 [7, i.e. instant $\left(x^{0}=0\right)$, front $\left(x^{+}=x^{0}+x^{3}=0\right)$, point $\left(x_{\mu} x^{\mu}=a^{2}>0, x^{0}>0\right)$, the dimenson of stability group which leaves the hypersurface of corresponding time invariant is the largest (i.e. seven out of the ten Poincare generators) in the front form which we call the LFD. Since the number of kinematic operators is maximum among the pos- sible forms of Hamiltonian dynamics, to a certain extent LFD is like sweeping dirt to a corner so that it leaves the rest of space clean 13 14. More reasons for our sweeping analogy may be due to the energy-momentum dispersion relation in LFD is rational; i.e.

$p^{-}=\frac{\vec{p}_{\perp}^{2}+m^{2}}{p^{+}}$,

where $p^{-}=p^{0}-p^{3}$ is the LF energy (the conjugate variable of the LF time $x^{+}=x^{0}+x^{3}$ ) and $p^{+}=p^{0}+p^{3}$ is the longitudinal LF momentum orthogonal to $p^{-}$for the particle with mass $m$ (or $p^{\mu} p_{\mu}=m^{2}$ ). From Eq. (11), it is very clear that the signs of $p^{+}$and $p^{-}$are correlated. Thus, for all the particles and antiparticles involved in the physical process, the LF longitudinal momentum cannot be negative,i.e. $p^{+} \geq 0$ in the LFD. This leads to the simplicity of LF vacuum, i.e. the quantum fluctuation of the vacuum is suppressed in LFD, except the zero-mode participation when all the particles and antiparticles in the physical process have zero LF longitudinal momenta, i.e. $p^{+}=0$ for all individual constituents.

The simple vacuum except the zero-modes is a remarkable achievement in LFD. This is indeed like sweeping dirt to a corner 1314 since the complexity of the vacuum is condensed to the zero-mode contribution of the quantum states while the rest of the vacuum is simple. Among the various successful applications of LFD utilizing the simplicity of vacuum, perhaps the most well-known example may be the parton model in deep inelastic lepton hadron scattering appreciated widely since 1970's.

However, the apparent simplicity of the LF vacuum yields a difficulty in understanding the novel phenomena associated with nontrivial vacua such as the spontaneous symmetry breaking, Higgs mechanism, chiral symmetry breaking, axial anomaly, $\theta$-vacuum, etc.. To understand these phenomena in LFD, we think that it is also very important to look into dirts piled at the corner in our sweeping analogy. First step in this direction would be to look for the zero-mode contributions in the vacuum phenomena. Realization of axial anomaly in LFD discussed in Ref.15] was an attempt to this direction. In the next section 
(Section 3), we discuss an aspect of the vector anomaly in the Standard Model and present a concrete example of a zero-mode contribution to the vector anomaly.

\section{Vector Anomaly in Standard Model}

Anomalies betray the true quantal character of a quantized field theory. Because they are invariably associated with divergent amplitudes, their evaluation has proven to be complicated, at times even leading to enigmatic results [16]. Nowadays there exists a vast literature on the subject and perhaps a consensus has been reached [17. By definition an anomaly is a radiative correction that violates a symmetry of the classical Lagrangian and usually involves counting infinities whether it is due to ultraviolet infinities or an infinite number of degrees of freedom [18]. As this breaking of symmetry may bring quantized theory in agreement with experiment, or, on the contrary spoil the renormalizability of the theory, Jackiw 18 discerns with this distinction in mind two types of infinities: good infinities and bad infinities.

In this presentation, we are concerned with the bad infinities which cause the anomalies that ultimately spoil the predictive power of the theory. In particular, we revisit the vector anomaly which led to the discussions of the requirement of adding a contact term to the magnetic moment and the superconvergence relations [19, etc., in an effort to rescue the theory long time ago. A brief historical remark on the anomaly associated with the fermion-triangle loop was made in Ref. 14.

The Lorentz-covariant and gauge-invariant CPeven electromagnetic $\gamma W^{+} W^{-}$vertex is defined 2021 by

$$
\begin{aligned}
\Gamma_{\alpha \beta}^{\mu}= & i e\left\{A\left[\left(p+p^{\prime}\right)^{\mu} g_{\alpha \beta}+2\left(g_{\alpha}^{\mu} q_{\beta}-g_{\beta}^{\mu} q_{\alpha}\right)\right]\right. \\
& +(\Delta \kappa)\left(g_{\alpha}^{\mu} q_{\beta}-g_{\beta}^{\mu} q_{\alpha}\right) \\
& \left.+\frac{\Delta Q}{2 M_{W}^{2}}\left(p+p^{\prime}\right)^{\mu} q_{\alpha} q_{\beta}\right\}
\end{aligned}
$$

where $p\left(p^{\prime}\right)$ is the initial(final) four-momentum of the $W$ gauge boson and $q=p^{\prime}-p$. Here, $\Delta \kappa$ and $\Delta Q$ are the anomalous magnetic and quadrupole moments, respectively. At tree level,

$A=1, \quad \Delta \kappa=0, \quad \Delta Q=0$,

for any $Q^{2}=-q^{2}$ because of the point-like nature of $W^{ \pm}$gauge bosons. Beyond the tree level, however,

$$
\begin{gathered}
A=F_{1}\left(Q^{2}\right), \quad-\Delta \kappa=F_{2}\left(Q^{2}\right)+2 F_{1}\left(Q^{2}\right), \\
-\Delta Q=F_{3}\left(Q^{2}\right),
\end{gathered}
$$

where $F_{1}, F_{2}$ and $F_{3}$ are the usual electromagnetic form factors for the spin-1 particles 224 . The physical form factors, charge $\left(G_{C}\right)$, magnetic $\left(G_{M}\right)$, and quadrupole $\left(G_{Q}\right)$, are also related in a well-known way to the form factors $F_{1}, F_{2}$ and $F_{3}[224]$.

Using the usual manifestly covariant technique of the dimensional regularization(DR) with $D=$ $4-\epsilon$, which we denote as DR4, we obtain:

$$
\begin{gathered}
\left(F_{2}\left(q^{2}\right)+2 F_{1}\left(q^{2}\right)\right)_{\mathrm{DR} 4}=\frac{g^{2} Q_{f}}{4 \pi^{2}}\left\{\int_{0}^{1} d x \int_{0}^{1-x} d y\right. \\
\left.\left[(2-3 x-3 y) \ln C^{2}\left(m_{1}, m_{1}\right)+\frac{2 f_{1}^{0}+f_{2}^{0}}{2 C^{2}\left(m_{1}, m_{1}\right)}\right]\right\}, \\
\left(F_{3}\left(q^{2}\right)\right)_{\mathrm{DR} 4}=-\frac{g^{2} Q_{f}}{4 \pi^{2}} \int_{0}^{1} d x \int_{0}^{1-x} d y \\
\frac{8 x y(x+y-1) M_{W}^{2}}{C^{2}\left(m_{1}, m_{1}\right)}
\end{gathered}
$$

where $C^{2}\left(m_{a}, m_{b}\right)=x m_{a}^{2}+y m_{b}^{2}+(1-x-y) m_{2}^{2}-$ $(x+y)(1-x-y) M_{W}^{2}-x y q^{2}, f_{1}^{0}=-2[(x+y)(1-$ $\left.x-y)^{2} M_{W}^{2}+(2-x-y) x y q^{2}+(x+y) m_{1}^{2}\right]$ and $f_{2}^{0}=2(x+y)\left[\left\{1-(x+y)^{2}\right\} M_{W}^{2}+x y q^{2}+m_{1}^{2}\right]$. Here, $m_{1}$ and $m_{2}$ are the masses of the charged fermion struck by the photon and the spectator fermion, respectively. The charge factor $Q_{f}$ includes the color factor $N_{c}$ if the fermion loop is due to the quark and $g^{2}=G_{F} M_{W} / \sqrt{2}$ in the SM.

The singular part involving $1 / \epsilon$ of $\left(F_{2}+2 F_{1}\right)_{\text {DR } 4}$ vanishes as expected and $F_{3}$ needs no regularization. From Eq. (5), we recover the well known $q^{2}=0$ results of the fermion-loop contribution to the physical quantities $\Delta \kappa$ and $\Delta Q$ 20 21]:

$$
\begin{aligned}
(\Delta \kappa)_{f} & =\frac{g^{2} Q_{f}}{4 \pi^{2}}\left[I_{4}+(F-2) I_{3}+(2 \Delta-\mathcal{E}) I_{2}\right] \\
(\Delta Q)_{f} & =\frac{g^{2} Q_{f}}{4 \pi^{2}} \frac{4}{3}\left[I_{3}-I_{4}\right]
\end{aligned}
$$


where

$I_{m}=\int_{0}^{1} d t \frac{t^{m}}{t^{2}-t F+\mathcal{E}}$,

and $F=1+\mathcal{E}-\Delta, \mathcal{E}=\frac{m_{2}^{2}}{M_{W}^{2}}, \Delta=\frac{m_{1}^{2}}{M_{W}^{2}}$.

In order to obtain the form factors other than by the standard DR method, we may use the Pauli-Villars regularization method (PVR) or the smearing method (SMR), which introduces fictitious particles with mass $\Lambda$. In the PVR, one replaces the amplitude $G^{\mu}\left(m_{1}, m_{2}\right)$ by the amplitude $G_{\mathrm{PV} 1}^{\mu}=G^{\mu}\left(m_{1}, m_{2}\right)-G^{\mu}\left(\Lambda_{1}, m_{2}\right)$ or $G_{\mathrm{PV} 2}^{\mu}=G^{\mu}\left(m_{1}, m_{2}\right)-G^{\mu}\left(m_{1}, \Lambda_{2}\right)$, depending on whether one chooses to replace the propagator of the charged fermion that is coupled to the photon or the spectator fermion by the combination $(\not p-m)^{-1}-(\not p-\Lambda)^{-1}$. We denote these regularization methods as PV1 and PV2, respectively. The smearing (SMR) procedure 23] consists in replacing the photon-vertex $\gamma^{\mu}$ by the vertex $S_{\Lambda}\left(p^{\prime}\right) \gamma^{\mu} S_{\Lambda}(p)$ using the smearing function $S_{\Lambda}(p)=\Lambda^{2} /\left(p^{2}-\Lambda^{2}+i \varepsilon\right)$.

The anomalous quadrupole moment $\Delta Q$ (or $\left.F_{3}\left(Q^{2}\right)\right)$ is found completely independent from the regularization methods as it must be, i.e.

$$
\left(F_{3}\right)_{\mathrm{SMR}}=\left(F_{3}\right)_{\mathrm{PV} 1}=\left(F_{3}\right)_{\mathrm{PV} 2}=\left(F_{3}\right)_{\mathrm{DR} 4} .
$$

However, we find that the anomalous magnetic moment $\Delta \kappa$ (or $\left.F_{2}\left(Q^{2}\right)+2 F_{1}\left(Q^{2}\right)\right)$ differs by some fermion-mass-independent constants depending on the regularization methods:

$$
\begin{gathered}
\left(F_{2}+2 F_{1}\right)_{\mathrm{SMR}}-\left(F_{2}+2 F_{1}\right)_{\mathrm{DR} 4}=\frac{g^{2} Q_{f}}{4 \pi^{2}}\left(\frac{1}{6}\right), \\
\left(F_{2}+2 F_{1}\right)_{\mathrm{PV} 1}-\left(F_{2}+2 F_{1}\right)_{\mathrm{DR} 4}=\frac{g^{2} Q_{f}}{4 \pi^{2}}\left(\frac{2}{3}\right), \\
\left(F_{2}+2 F_{1}\right)_{\mathrm{PV} 2}-\left(F_{2}+2 F_{1}\right)_{\mathrm{DR} 4}=\frac{g^{2} Q_{f}}{4 \pi^{2}}\left(-\frac{1}{3}\right)
\end{gathered}
$$

These fermion-mass-independent differences are the vector anomalies that we pointed out 24 . Unless they are completely cancelled, they would make a unique prediction of $\Delta \kappa$ impossible. Within the SM, they are completely cancelled due to the zero-sum of the charge factor $\left(\sum_{f} Q_{f}=0\right)$ in each generation.

Since most calculations in the previous literature 1925 were limited essentially to use the manifestly covariant dimensional regularization method, it has not yet been so clear how the appearance of the vector anomaly changes in LF Hamiltonian formulation. In LFD, the Wick rotation to Euclidean space can never be used and the divergent integrations are given only over the perpendicular components $\left(k_{x}, k_{y}\right)$ of the integration variable after performing the LF-energy $\left(k^{-}\right.$ pole) integration in Minkowski space. The infinite parts in $D=2-\epsilon$ can be expressed in the familiar form containing $1 / \epsilon$, which in the PVR and the SMR is replaced by a form involving $\ln \Lambda$. We denote the LF regularization with $D=2-\epsilon$ as DR2 for the distinction from the manifestly covariant DR4 regularization. The details of the calculation were presented in Ref. 24, and in this presentation we note only the features drastically different from the manifestly covariant case.

In LFD, we compute the form factors using the following relation in the $q^{+}=q^{0}+q^{3}=0$ frame,

$$
\begin{aligned}
G_{++}^{+} & =2 p^{+}\left(F_{1}+\eta F_{3}\right), \\
G_{+0}^{+} & =p^{+} \sqrt{2 \eta}\left(2 F_{1}+F_{2}+2 \eta F_{3}\right), \\
G_{+-}^{+} & =-2 p^{+} \eta F_{3}, \\
G_{00}^{+} & =2 p^{+}\left(F_{1}-2 \eta F_{2}-2 \eta^{2} F_{3}\right),
\end{aligned}
$$

where $\eta=Q^{2} / 4 M_{W}^{2}$. $G_{+-}^{+}$depends on $F_{3}$ only and $G_{++}^{+}$involves only $F_{1}$ and $F_{3}$. Therefore, the simplest procedure is to solve first for $F_{3}$ from $G_{+-}^{+}$. Next, $F_{1}$ is obtained from $G_{++}^{+}$and $F_{3}$. Finally, $F_{2}$ can be obtained from the other matrix elements. The two relevant choices are to use either $G_{+0}^{+}$or $G_{00}^{+}$and consequently we may define

$$
\begin{gathered}
\left(F_{2}+2 F_{1}\right)^{+0}=\frac{1}{p^{+}}\left[\frac{G_{+0}^{+}}{\sqrt{2 \eta}}+G_{+-}^{+}\right], \\
\left(F_{2}+2 F_{1}\right)^{00}=\frac{1}{4 p^{+} \eta}\left[(1+2 \eta) G_{++}^{+}-G_{00}^{+}\right. \\
\left.+(1+4 \eta) G_{+-}^{+}\right] .
\end{gathered}
$$

Splitting the covariant fermion propagator into the LF-propagating part and the LFinstantaneous part, the divergences can show up both in the valence amplitude containing only the LF-propagating fermions and in the nonvalence amplitude containing a LF-instantaneous fermion. In the $q^{+}=0$ frame, one might expect that the non-valence contribution is absent since the integration range for the non-valence amplitude diminishes to zero. However, this is not the 
case as we pointed out in Ref. 4. Calling the nonzero contribution from the non-valence part in the $q^{+}=0$ frame the zero-mode, we find that only the helicity zero-to-zero amplitude $G_{00}^{+}$receives a zero-mode contribution and the non-vanishing zero-mode contribution to $G_{00}^{+}$is given by

$$
\begin{gathered}
\left(G_{00}^{+}\right)_{\text {z.m. }}=\frac{g^{2} Q_{f} p^{+}}{2 \pi^{3} M_{W}^{2}} \int_{0}^{1} d x \int d^{2} \vec{k}_{\perp} \\
\frac{\vec{k}_{\perp}^{2}+m_{1}^{2}-x(1-x) Q^{2}}{\vec{k}_{\perp}^{2}+m_{1}^{2}+x(1-x) Q^{2}} \neq 0 .
\end{gathered}
$$

The zero-mode contribution to $G_{00}^{+}$is crucial because the unwelcome divergences from the valence part due to the terms with a power of the transverse momentum such as $\left(k_{\perp}^{2}\right)^{2}$ are precisely cancelled by the same terms with the opposite sign from the zero-mode contribution. Neither such high power $\left(k_{\perp}^{2}\right)^{2}$ term nor the zero-mode contribution exist in $G_{+0}^{+}$. The essential results directly related to the vector anomalies in DR2 are summarized as follows:

$$
\begin{gathered}
\left(F_{2}+2 F_{1}\right)_{\mathrm{DR} 2}^{+0}-\left(F_{2}+2 F_{1}\right)_{\mathrm{DR} 4}=\frac{g^{2} Q_{f}}{4 \pi^{2}}\left(\frac{1}{6}\right) \\
\left(F_{2}+2 F_{1}\right)_{\mathrm{DR} 2}^{00}-\left(F_{2}+2 F_{1}\right)_{\mathrm{DR} 4}= \\
-\frac{g^{2} Q_{f}}{4 \pi^{2}}\left(\frac{1}{2 \eta}\right)\left(\frac{1}{3}+\frac{2 \eta}{9}\right) .
\end{gathered}
$$

The fact that $\left(F_{2}+2 F_{1}\right)_{\mathrm{DR} 2}^{+0}$ and $\left(F_{2}+2 F_{1}\right)_{\mathrm{DR} 2}^{00}$ disagree indicates that the symptom of vector anomaly in DR2 appears as the violation of the rotation symmetry or the angular momentum conservation (i.e. angular condition 26]). This appearance is drastically different from the case of the manifestly covariant calculation. However, the anomaly-free condition $\left(\sum_{f} Q_{f}=0\right)$ in the $\mathrm{SM}$ again removes the difference and restores the rotation symmetry and the angular momentum conservation in LFD.

Besides DR2, we have also applied other regularization methods in LFD, such as PV1, PV2 and SMR, which carry an explicit cutoff parameter $\Lambda$. Interestingly, in each of these regularization methods, we find that not only $\left(F_{2}+2 F_{1}\right)^{+0}=$ $\left(F_{2}+2 F_{1}\right)^{00}$ but also the LF result completely agrees with the corresponding manifestly covariant result: e.g.

$$
\left(F_{2}+2 F_{1}\right)_{\mathrm{PV} 1}^{+0}=\left(F_{2}+2 F_{1}\right)_{\mathrm{PV} 1}^{00}=\left(F_{2}+2 F_{1}\right)_{\mathrm{PV} 1}^{c o v},
$$

where $\left(F_{2}+2 F_{1}\right)_{\mathrm{PV} 1}^{\text {cov }}$ is the result shown in Eq. (9).
This proves that the rotation symmetry is not violated in the regularization methods with an explicit cutoff $\Lambda$ unlike the above DR2 case. However, we note that the zero-mode contribution in $\left(F_{2}+2 F_{1}\right)^{00}$ is crucial to get Eq. (14). The details of our calculations including an interesting consequence in the PV2 case where the zero-mode is artificially removed, were presented in Ref. 24]. In these LF regularization methods, we find that the vector anomalies occur just the same way as in the manifestly covariant case (see Eq. (9)). Within the SM, they are again completely cancelled due to the zero-sum of the charge factor $\left(\sum_{f} Q_{f}=0\right)$ in each generation.

\section{Power Counting Method}

For hadron phenomenology, Jaus [8] and we [4 91011 independently investigated the spin-1 electroweak form factors in the past few years. As mentioned in the introduction, Jaus 8] proposed a covariant LF approach involving the lightlike four vector $\omega^{\mu}\left(\omega^{2}=0\right)$ as a variable and developed a way of finding the zero-mode contribution to remove the spurious amplitudes proportional to $\omega^{\mu}$. Our formulation, however, is intrinsically distinguished from this $\omega$-dependent formulation since it involves neither $\omega^{\mu}$ nor any unphysical form factors. Our method of finding the zero-mode contribution is a direct powercounting of the longitudinal momentum fraction in the $q^{+} \rightarrow 0$ limit for the off-diagonal elements in the Fock-state expansion of the current matrix 491011 . Since the longitudinal momentum fraction is one of the integration variables in the LF matrix elements (i.e. helicity amplitudes), our power-counting method is straightforward as far as we know the behaviors of the longitudinal momentum fraction in the integrand.

For a rather simple (manifestly covariant) vertex $\Gamma^{\mu}=\gamma^{\mu}$, both Jaus and we agree on the absence of zero-mode contributions to the spin1 electroweak form factors. However, Jaus and we do not agree when $\Gamma^{\mu}$ is extended to the more phenomenologically accessible ones given by

$\Gamma^{\mu}=\gamma^{\mu}-\frac{\left(k+k^{\prime}\right)^{\mu}}{D}$,

where $k$ and $k^{\prime}$ are the relative four momenta for 
the two constituent quarks. Although Jaus's calculation and our calculation used the same denominator $D$ in Eq.(15), they led to different conclusions in the analysis of the zero-mode contribution. Even if $D$ is chosen in such a way to get the manifestly covariant $\Gamma^{\mu}$, the difference in the conclusions doesn't go away.

For the spin-1 elastic form factor calculations, Jaus's conclusion 8 was that the matrix elements $<h^{\prime}=0\left|J^{+}\right| h=1>$ and $<h^{\prime}=0\left|J^{+}\right| h=$ $0>$ both get zero-mode contributions, so that one cannot avoid the zero-mode contributions to the form factor $F_{2}\left(q^{2}\right)$ for the vector meson. However, we found that only the matrix element $<h^{\prime}=$ $0\left|J^{+}\right| h=0>$ gets a zero-mode contribution so that we can avoid the zero-mode contribution to $F_{2}\left(q^{2}\right)$ if we do not use the matrix element $<h^{\prime}=$ $0\left|J^{+}\right| h=0>[10$.

Similarly, for the weak transition form factors between the pseudoscalar $(\mathrm{P})$ and vector $(\mathrm{V})$ mesons, Jaus 8 concluded that the form factor $A_{1}\left(q^{2}\right)$ [or $f\left(q^{2}\right)$ ] receives a zero-mode contribution. We again do not agree with his result but find that $f\left(q^{2}\right)$ is free from the zero-mode contribution if the denominator $D$ in Eq. (15) contains the term proportional to the LF energy $\left(k^{-}\right)^{n}$ with the power $n>0$. The phenomenologically accessible LFQM satisfies this condition $n>0$.

As we have shown in detail in Ref. 11], we can determine the existence/nonexistence of the zeromode contribution to $f\left(q^{2}\right)$ by counting the factors of the longitudinal momentum fraction. For example, if $D=D_{\text {cov }}(k \cdot P) \equiv\left[2 k \cdot P+M_{V}\left(m_{q}+\right.\right.$ $\left.\left.m_{\bar{q}}\right)-i \epsilon\right] / M_{V}$, where $P$ is the four momentum of the vector meson 27, then $D$ contains a term proportional to the LF energy $\left(k^{-}\right)^{n}$ with the power $n=1$. This power-counting shows that the form factor $f\left(q^{2}\right)$ should not receive a zero-mode contribution in the $D_{\text {cov }}(k \cdot P)$ case. When the manifestly covariant model for the vector meson vertex $\Gamma^{\mu}$ is available, we have confirmed that the results found our way coincide with the ones from the manifestly covariant calculation.

As we show in Fig 1 our result $f^{\text {ours }}$ (circle) obtained in the $q^{+}=0$ frame (or Drell-Yan frame so that $f^{\text {ours }}=f^{\text {DY }}$ ) is in an exact agreement with the full result (solid line) in the purely longitudinal $q^{+}>0$ frame 11]. This agreement as-

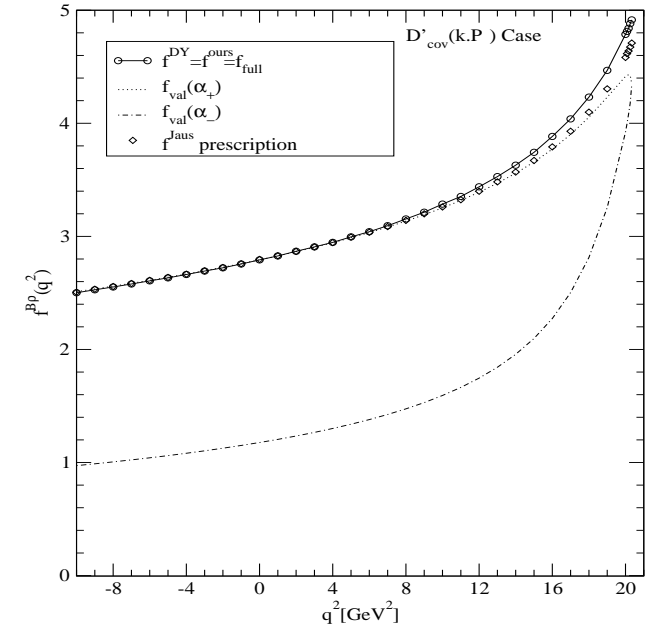

Figure 1. Weak form factor $f\left(q^{2}\right)$ for $B \rightarrow \rho$ transition in the case of the vector meson vertex with $D_{\text {cov }}(k \cdot P)$.

sures that our result is correct and there is no zero-mode contribution to $f\left(q^{2}\right)$ for the vertex with $D_{\text {cov }}(k \cdot P)$. The nonvalence contributions in the purely longitudinal frame can be found as the difference between the full result and $f_{\mathrm{val}}\left(\alpha_{+}\right)$ (dotted line) or $f_{\text {val }}\left(\alpha_{-}\right)$(dotted-dashed line) depending on the recoil direction of $B$-meson after it decays into $\rho$ and leptons.

Comparing Jaus's result with ours, however, we find that $f^{\text {ours }}$ and $f^{\text {Jaus }}$ differ about $4 \%$ at $q=q_{\max }$ although they coincide at $q^{2}=0$. This difference between Jaus's and ours shows that Jaus's conclusion for the existence of zero-mode in $f\left(q^{2}\right)$ doesn't apply in this case $D=D_{\text {cov }}(k \cdot P)$. Thus, Jaus's method of finding zero-mode contributions has a limitation in the choice of $\Gamma^{\mu}$.

\section{Conclusions}

In this presentation, we discussed a concrete example of a zero-mode contribution in the Standard Model analysis of the vector anomaly computing the CP-even form factors of the $W^{ \pm}$gauge bosons. The vector anomaly in the fermiontriangle-loop is real and shows a non-vanishing 
LF zero-mode contribution to the helicity zero-tozero amplitude $G_{00}^{+}$. In LFD, the helicity dependence of the vector anomaly exhibits a violation of Lorentz symmetry. This may be contrasted with the manifestly covariant Lagrange formulation where the same anomaly appears as a violation of gauge symmetry. Our findings in this work may provide a bottom-up fitness test not only to the LFD calculations but also to the theory itself, whether it is an extension of the Standard Model or an effective field theory of composite systems.

In the absence of zero-mode contributions, the hadron form factors can be obtained rather straightforwardly by just taking into account only the valence contributions. Moreover, the Lorentz covariance of the result can be assured in the absence of zero-mode contributions. For successful hadron phenomenology, it is thus significant to pin down which physical observables receive nonvanishing zero-mode contribution. Our powercounting-method discussed in this work seems to provide a correct way of pinning down the existence/nonexistence of the zero-mode contribution to physical observables.

\section{REFERENCES}

1. See, e.g., K. Goeke, M. V. Polyakov and M. Vanderhaeghen, Prog. Part. Nucl. Phys. 47 (2001) 401, and references therein.

2. For a review of B-Physics with emphasis on LFD, see C.-R. Ji and H.-M.Choi, Nucl. Phys. Proc. Suppl. 90 (2000) 93.

3. An example of applying LF wavefunctions to RHIC physics can be seen in R. J. Fries, B. Müller, C. Nonaka and S. A. Bass, Phys. Rev. C68 (2003) 044902.

4. B. L. G. Bakker, H.-M. Choi and C.-R. Ji, Phys. Rev. D65 (2002) 116001.

5. B. L. G. Bakker and C.-R. Ji, Phys. Rev. D62 (2000) 074014.

6. B. L. G. Bakker, M. A. DeWitt, C.-R.Ji and Y. Mishchenko, Phys. Rev. D (2005) in press.

7. P. A. M. Dirac, Rev. Mod. Phys. 21 (1949) 392.

8. W. Jaus, Phys. Rev. D60 (1999) 054026; Phys. Rev. D67 (2003) 094010.

9. B. L. G. Bakker, H.-M. Choi and C.-R. Ji,
Phys. Rev. D67 (2003) 113007.

10. H.-M. Choi and C.-R. Ji, Phys. Rev. D70 (2004) 053015.

11. H.-M. Choi and C.-R. Ji, Phys. Rev. D72 (2005) 013004.

12. J. P. C. de Melo, J. H. Sales, T. Frederico and P. U. Sauer, Nucl. Phys. A631 (1998) 574C; H.-Y. Cheng, C.-K. Chua and C.-W. Hwang, Phys. Rev. D69 (2004) 074025.

13. C.-R. Ji, IPPP/03/71, pp.86-89, Durham 2003; Nucl. Phys. A737 (2004) 255.

14. C.-R. Ji and B. L. G. Bakker, Few-Body Systems 36 (2005) 137.

15. C.-R. Ji and S.-J. Rey, Phys. Rev. D53 (1996) 5815.

16. D.G. Sutherland, Nucl. Phys. B2 (1967) 433; M. Veltman, Proc. Royal Soc. A30 (1967) 107.

17. R.A. Bertlmann, Anomalies in Quantum Field Theory, (Clarendon Press, Oxford, 1996).

18. R. Jackiw, 'The unreasonable effectiveness of quantum field theory', in T.Y. Cao (ed.), Conceptual foundations of quantum field theory, Cambridge UP, (Cambridge, 1999).

19. L. DeRaad, K. Milton and W. Tsai, Phys. Rev. D9 (1974) 2847; Phys. Rev. D12 (1975) 3972 .

20. W. A. Bardeen, R. Gastmans and B. Lautrup, Nucl. Phys. B46 (1972) 319.

21. G. Couture and J. N. Ng, Z. Phys. C35 (1987) 65.

22. B. L. G. Bakker and C.-R. Ji, Phys. Rev. D65 (2002) 073002.

23. B. L. G. Bakker, H.-M. Choi, and C.-R. Ji, Phys. Rev. D63 (2001) 074014.

24. B. L. G. Bakker and C.-R. Ji, Phys. Rev. D71 (2005) 053005.

25. For a review on the $\gamma W^{+} W^{-}$vertex within the effective Lagrangian approach, see J. Ellison and J. Wudka, Annu. Rev. Nucl. Part. Sci. 48 (1998) 33, and references therein. See also, S. Weinberg, Eur. Phys. J. C34 (2004) 5.

26. C.Carlson and C.-R.Ji, Phys. Rev. D67 (2002) 116002

27. J. P. C. de Melo and T. Frederico, Phys. Rev. C55 (1997) 2043. 